C. DAI AND S. WANG

KODAI MATH. J.

10 (1987), 292-297

\title{
ON NEVANLINNA'S INVERSE PROBLEM OF FINITE ORDER
}

\author{
By Chong-Ji Dai AND Shu-Pei Wang
}

\section{Introduction.}

In this paper we solved one conjecture of W. K. Hayman ([10], problem 1.29). We obtained the following

THEOREM. Let $\left\{a_{n}\right\}_{n=1}^{N}$ be a preassigned sequence of finite complex numbers and $\left\{\delta_{n}\right\}_{n=1}^{N}(N \leqq \infty)$ be a sequence of positive numbers, $0<\delta_{n} \leqq 1 \quad(1 \leqq n \leqq N)$, $0<\lambda<\infty$. If

$$
\sum_{n=1}^{N} \delta_{n}^{1 / 3} \leqq A(\lambda), \quad \text { here } \quad A(\lambda)= \begin{cases}(8 \lambda)^{-1 / 3} & 1 \leqq \lambda<\infty \\ 1 / 2(\lambda)^{2 / 3} & 1 / 4 \leqq \lambda<1 \\ \lambda(1-2 \lambda)^{1 / 3} & 0<\lambda<1 / 4 .\end{cases}
$$

Then there exists a meromorphic function $f(z)$ of order $\lambda$, and $\delta\left(a_{n}, f\right) \geqq \delta_{n}$ for any $1 \leqq n \leqq N$. (Here $\delta(a, f)$ denotes the Nevanlinna-deficiency.)

In 1929, R. Nevanlinna [1] proposed this problem: can we construct a meromorphic function $f(z)$ such that $f(z)$ has an arbitrary sequence $\left\{a_{n}\right\}$ of deficient values and no others and further that $\delta\left(a_{n}, f\right)=\delta_{n}$, where $\left\{\delta_{n}\right\}$ is an arbitrary sequence subject to $\Sigma \delta_{n} \leqq 2$ ? This is the Nevanlinna's inverse problem. Late himself [2], F.E. Ullrich [3], Thiem [4] and A. A. Goldberg [5] obtained some results in different cases. In 1962, W. H. J. Fuchs and W.K. Hayman [9] solved this problem in the case of entire function. Up to 1977, D. Drasin [6] solved this inverse problem completely. But the functions in the above results are of infinite order. As to the Nevanlinna inverse problem of finite order, A. A. Goldberg [7] and N.U. Arakelyan [8] have studied and obtained some results. Since the "deficiency-problem" of meromorphic function of finite order has not been solved completely, both of them only constructed this kind of finite order functions which have infinite numbers of deficient values, and nothing is known as for deficiency. However, Hayman [9] got this interesting result: if the given sequence of positive numbers $\left\{\delta_{n}\right\}$ satisfy $\sum \delta_{n}^{1 / 3} \leqq 9^{-1 / 3}$, then there exists a meromorphic function $f(z)$ of order 1 , and $\delta\left(a_{n}, f\right) \geqq \delta_{n}(1 \leqq n \leqq N)$. Later, he gave this conjecture [10]: in the condition $\sum \delta_{n}^{1 / 3} \leqq 9^{-1 / 3}$, can we have $A(\lambda)(0<\lambda<\infty)$ substituted for $9^{-1 / 3}$ and construct a

Received January 20, 1987. 
meromorphic function of order $\lambda$ and $\delta\left(a_{n}, f\right) \geqq \delta_{n}(1 \leqq n \leqq N)$ ? In this present paper, we shall be affirm his conjecture and have $A(\lambda)$ as in Theorem.

The authors would like to thank the referee for his valuable comments.

\section{Lemmas.}

LEMMA 1. Let $\left\{a_{n}\right\}_{n=1}^{N}$ be an arbitrary sequence of finite complex numbers and $\left\{\eta_{n}\right\}_{n=1}^{N}$ be an arbitrary sequence of positive numbers, satisfying $0<\eta_{n} \leqq 1$, $\sum_{n=1}^{N} \eta_{n} \leqq 1, \lambda$ be an arbitrary real number, $1 \leqq \lambda<\infty$. Then there exists a meromorphic function $f(z)$ of order $\lambda$ and $\delta\left(a_{n}, f\right) \geqq \frac{1}{8 \lambda} \eta_{n}^{3}$.

Proof. Without loss of generality, we assume $\left\{\eta_{n}\right\}$ be monotone decreasing. Let $\eta_{0}=\eta_{1}, \theta_{0}=0, \theta_{n}=\left(\sum_{\nu=0}^{n-1} \eta_{\nu}\right) \pi, \quad(n=1,2, \cdots, N)$. So $\left\{\theta_{n}\right\}_{n=0}^{N}$ is increasing strictly and

$$
\lim _{n \rightarrow N} \theta_{n}=\pi \sum_{\nu=0}^{N-1} \eta_{\nu} \leqq 2 \pi
$$

By choosing a suitable sequence $\left\{C_{n}\right\}$ of positive numbers, such that

$$
S_{1}=\sum_{n=1}^{N} C_{n}\left|a_{n}\right|<\infty, \quad S_{2}=\sum_{n=1}^{N} C_{n}<\infty .
$$

Let $f(z)=\frac{\phi_{1}(z)}{\phi_{2}(z)}$, where

$$
\phi_{1}(z)=\sum_{n=1}^{N} C_{n} a_{n} \exp \left(z^{\lambda} e^{-i \theta_{n}}\right) ; \quad \phi_{2}(z)=\sum_{n=1}^{N} C_{n} \exp \left(z^{\lambda} e^{-i \theta_{n}}\right) .
$$

Notice that if $|z| \leqq r$, then

$$
\left|\phi_{1}(z)\right| \leqq \sum_{n=1}^{N} C_{n}\left|a_{n}\right| e^{r^{\lambda}}=S_{1} e^{r^{\lambda}} ; \quad\left|\phi_{2}(z)\right| \leqq \sum_{n=1}^{N} C_{n} e^{r^{\lambda}}=S_{2} e^{r^{\lambda}}
$$

So $\phi_{1}(z), \phi_{2}(z)$ are entire functions. So $f(z)$ is a meromorphic function and

$$
T(r, f) \leqq T\left(r, \phi_{1}\right)+T\left(r, \phi_{2}\right) \leqq 2 r^{\lambda}+O(1) .
$$

For each $n$, we put

$$
I_{n}=\left[\frac{1}{\lambda}\left(\theta_{n}-\frac{1}{4} \pi \eta_{n}\right), \frac{1}{\lambda}\left(\theta_{n}+\frac{1}{4} \pi \eta_{n}\right)\right] .
$$

Since

$$
\begin{aligned}
& \theta_{n}-\frac{1}{4} \pi \eta_{n}=\pi\left\{\sum_{\nu=0}^{n-2} \eta_{\nu}+\eta_{n-1}-\frac{1}{4} \eta_{n}\right\}>\theta_{n-1}>0, \\
& \theta_{n}+\frac{1}{4} \pi \eta_{n} \leqq \theta_{n+1} \leqq 2 \pi,
\end{aligned}
$$

and $\lambda \geqq 1$, so we have $I_{n} \subseteq[0,2 \pi]$. Let $\theta \in I_{n}$, 


$$
\begin{aligned}
& \lambda \theta-\theta_{\nu} \geqq\left(\theta_{n}-\frac{1}{4} \pi \eta_{n}\right)-\theta_{n-1}=\pi\left(\eta_{n-1}-\frac{1}{4} \eta_{n}\right) \geqq \frac{3}{4} \pi \eta_{n}, \quad \text { if } \nu<n ; \\
& \theta_{\nu}-\lambda \theta \geqq \theta_{n+1}-\left(\theta_{n}+\frac{1}{4} \pi \eta_{n}\right)=\frac{3}{4} \pi \eta_{n}, \quad \text { if } \nu>n .
\end{aligned}
$$

So, if $\theta \in I_{n}, \nu \neq n$, we have

$$
\left|\lambda \theta-\theta_{\nu}\right| \geqq \frac{3}{4} \pi \eta_{n}
$$

But $\left|\lambda \theta-\theta_{\nu}\right| \leqq \max _{\nu \geq 1}\left\{\left|\theta_{n-1}-\theta_{\nu}\right|,\left|\theta_{n+1}-\theta_{\nu}\right|\right\} \leqq \pi, \quad(\nu \neq n, \nu \geqq 1)$. By (6), hence we may have: for $\theta \in I_{n}, \nu \neq n, \nu \geqq 1$,

So

$$
\frac{3}{4} \pi \eta_{n} \leqq\left|\lambda \theta-\theta_{\nu}\right| \leqq \pi
$$

$$
\cos \left(\lambda \theta-\theta_{\nu}\right) \leqq \cos \frac{3}{4} \pi \eta_{n}, \quad\left(\theta \in I_{n}, \nu \neq n\right) .
$$

Now by (3), (7), for $\theta \in I_{n}$, we have

$$
\begin{aligned}
& \left|\phi_{1}(z)-C_{n} a_{n} \exp \left(z^{\lambda} e^{-i \theta_{n}}\right)\right|=\left|\sum_{\nu \neq n} C_{\nu} a_{\nu} \exp \left(z^{\lambda} e^{-i \theta_{\nu}}\right)\right| \\
& \leqq \sum_{\nu \neq n} C_{\nu}\left|a_{\nu}\right| \exp \left(r^{\lambda} \cos \left(\lambda \theta-\theta_{\nu}\right)\right) \leqq S_{1} \exp \left(r^{\lambda} \cos \frac{3}{4} \pi \eta_{n}\right) . \\
& \left|\phi_{2}(z)\right|=\left|C_{n} \exp \left(z^{\lambda} e^{-i \theta_{n}}\right)+\sum_{\nu \neq n} C_{\nu} \exp \left(z^{\lambda} e^{-i \theta_{\nu}}\right)\right| \\
& \geqq\left[C_{n} \exp \left(r^{\lambda} \cos \left(\lambda \theta-\theta_{n}\right)\right)-\sum_{\nu \neq n} C_{\nu}\left|\exp \left(z^{\lambda} e^{-i \theta_{\nu}}\right)\right|\right] \\
& \geqq C_{n} \exp \left(r^{\lambda} \cos \frac{\pi}{4} \eta_{n}\right)-S_{2} \exp \left(r^{\lambda} \cos \frac{3}{4} \pi \eta_{n}\right) \\
& >\frac{1}{2} C_{n} \exp \left(r^{\lambda} \cos \frac{1}{4} \pi \eta_{n}\right), \quad\left(r>r_{0}\right) .
\end{aligned}
$$

From (2), (6), (8), (9), for each $n$ and $r>r_{0}$, we have

$$
\begin{aligned}
\left|f(z)-a_{n}\right| & =\frac{\left|\phi_{1}(z)-a_{n} \phi_{2}(z)\right|}{\left|\phi_{2}(z)\right|} \\
& \leqq \frac{1}{\left|\phi_{2}(z)\right|}\left|\sum_{\nu \neq n} C_{\nu}\left(\left|a_{\nu}\right|+\left|a_{n}\right|\right)\right| \exp \left(z^{\lambda} e^{\left.-i \theta_{\nu}\right) \mid} \mid\right. \\
& \leqq \frac{\left\{S_{1}+\left|a_{n}\right| S_{2}\right\} \exp \left(r^{\lambda} \cos \frac{3}{4} \pi \eta_{n}\right)}{\frac{1}{2} C_{n} \exp \left(r^{\lambda} \cos \frac{1}{4} \pi \eta_{n}\right)} .
\end{aligned}
$$

So, as $r \rightarrow \infty, \theta \in I_{n}$, uniformly we have 


$$
\begin{aligned}
\log ^{+}\left|\frac{1}{f\left(r e^{i \theta}\right)-a_{n}}\right| & \geqq r^{2}\left(\cos \frac{1}{4} \pi \eta_{n}-\cos \frac{3}{4} \pi \eta_{n}\right)+O(1) \\
& =2 r^{2} \sin \frac{1}{4} \pi \eta_{n} \cdot \sin \frac{1}{2} \pi \eta_{n}+O(1) \\
& \geqq 2 r^{2} \cdot \frac{2}{\pi}\left(\frac{1}{4} \pi \eta_{n}\right) \cdot \frac{2}{\pi}\left(\frac{1}{2} \pi \eta_{n}\right)+O(1) \\
& =r^{2} \eta_{n}^{2}+O(1) .
\end{aligned}
$$

By (11), we have

$$
\begin{aligned}
m\left(r, \frac{1}{f-a_{n}}\right) & \geqq \frac{1}{2 \pi} \int_{I_{n}} \log ^{+} \frac{1}{\left|f\left(r e^{i \theta}\right)-a_{n}\right|} d \theta \\
& \geqq \frac{1}{4 \lambda} r^{\lambda} \eta_{n}^{3}+O(1) .
\end{aligned}
$$

By (4), (12), we have

$$
\begin{aligned}
\delta\left(a_{n}, f\right) & =\lim _{r \rightarrow \infty} \frac{m\left(r, \frac{1}{f-a_{n}}\right)}{T(r, f)} \\
& \geqq \varliminf_{r \rightarrow \infty} \frac{\frac{1}{4 \lambda} r^{2} \eta_{n}^{3}+O(1)}{2 r^{\lambda}+O(1)}=\frac{1}{8 \lambda} \eta_{n}^{3} .
\end{aligned}
$$

Since $T(r, f) \geqq m\left(r, \frac{1}{f-a_{n}}\right)+O(1) \geqq \frac{1}{4 \lambda} r^{\lambda} \eta_{n}^{3}+O(1)$ and by (4), hence we have that $f(z)$ is a meromorphic function of order $\lambda$, which complete our proof of Lemma 1.

LEMMA 2. Let $\left\{a_{n}\right\}_{n=1}^{N}$ as in Lemma $1, \lambda$ be an arbitrary positive number and $\frac{1}{4} \leqq \lambda<1,\left\{\eta_{n}\right\}_{n=1}^{N}$ be a sequence of positive numbers, satisfying $0<\eta_{n} \leqq \lambda$, $\sum_{n=1}^{N} \eta_{n} \leqq \lambda$. Then there exists a meromorphic function $f(z)$ of order $\lambda$ and $\delta\left(a_{n}, f\right)$ $\geqq \frac{1}{8 \lambda} \eta_{n}^{3}(1 \leqq n \leqq N)$.

Sketch of the proof. (The notation is the same as in Lemma 1) Notice that here $I_{n} \subseteq[0,2 \pi]$.

In fact, since $I_{n}=\left[\frac{1}{\lambda}\left(\theta_{n}-\frac{1}{4} \pi \eta_{n}\right), \frac{1}{\lambda}\left(\theta_{n}+\frac{1}{4} \pi \eta_{n}\right)\right]$ and $\theta_{n}+\frac{1}{4} \pi \eta_{n} \leqq \theta_{n+1}$ $\leqq 2 \pi \lambda$, so $\frac{1}{\lambda}\left(\theta_{n}+\frac{1}{4} \pi \eta_{n}\right) \leqq 2 \pi$, and obviously, $\theta_{n}-\frac{1}{4} \pi \eta_{n}>0$. Hence $I_{n} \leqq[0,2 \pi]$. The rest of the proof is the same as in Lemma 1 .

LEMMA 3. Let $\left\{a_{n}\right\}_{n=1}^{N}$ as in Lemma $1, \lambda$ be an arbitrary positive number 
and $0<\lambda<\frac{1}{4},\left\{\eta_{n}\right\}_{n=1}^{N}$ be a sequence of positive numbers, satisfyng $0<\eta_{n} \leqq \lambda$ and $\sum_{n=1}^{N} \eta_{n} \leqq \lambda$. Then there exists a meromorphic function $f(z)$ of order $\lambda$, and $\delta\left(a_{n}, f\right) \geqq(1-2 \lambda) \eta_{n}^{3}(1 \leqq n \leqq N)$.

Sketch of the proof. Here only let $I_{n}=\left[\frac{1}{\lambda}\left(\theta_{n}-\lambda \pi \eta_{n}\right), \frac{1}{\lambda}\left(\theta_{n}+\lambda \pi \eta_{n}\right)\right]$, it's easy to know $I_{n} \subseteq[0,2 \pi]$. The rest of the proof is similar to that of Lemma 1 .

\section{Proof of the theorem.}

Put $S=\sum_{n=1}^{N} \delta_{n}^{1 / 3}$, so $\sum_{n=1}^{N} \frac{\delta_{n}^{1 / 3}}{S}=1$, and put

$$
\begin{aligned}
& \eta_{n}=\frac{1}{S} \delta_{n}^{1 / 3}, \quad \text { if } \lambda \geqq 1, \\
& \eta_{n}=\frac{1}{S} \lambda \delta_{n}^{1 / 3}, \quad \text { if } 0<\lambda<1 .
\end{aligned}
$$

Then, from the above Lemmas, we have

$$
\delta\left(a_{n}, f\right) \geqq \begin{cases}\frac{1}{8 \lambda S^{3}} \delta_{n}, & 1 \leqq \lambda<\infty, \\ \frac{\lambda^{2}}{8 S^{3}} \delta_{n}, & \frac{1}{4} \leqq \lambda<1, \\ \frac{(1-2 \lambda) \lambda^{3}}{S^{3}} \delta_{n}, & 0<\lambda<\frac{1}{4} .\end{cases}
$$

So, as $\sum_{n=1}^{N} \delta_{n}^{1 / 3}=S \leqq A(\lambda)$, we have $\delta\left(a_{n}, f\right) \geqq \delta_{n}(1 \leqq n \leqq N)$, which complete the proof of our theorem.

\section{REFERENCES}

[1] R. Nevanlinna, Le théorème de Picard-Borel et la théorie des fonctions méromorphcs, Paris, 1929.

[2] R. Nevanlinna, Über Riemannschan Flöchan mit endlich Vielen, Windangspankten, Acta Math. 58 (1932), 295-373.

[3] F.E. UllRich, Zum Umkehrproblem der Wertvertelungslehre, Nachr. Fes. Wiss. Göttingen Math.-Plys. K. L., No. 9 (1936).

[4] Le-VAN Thiem, Uber das Umkehrproblem der Vestverteilunglhre. Comment. Math. Helv. 23 (1949), 26-49.

[5] A. A. Goldberg AND I. V. OstrovskiI, The Distribution of values of Meromorphic Functions (Russian), Nauka, Moscow 1970.

[6] D. Drasin, The Inverse Problem of the Nevanlinna Theorem, Acta Math. 128 (1977), 81-151.

[7] A. A. Goldberg, Ukrain Mut. Zh. 11 (1959), 438-443.

[8] N.U. ARakelyan, Доклады Академии Наук, Том 170 (1966), 999-1002. 
[9] W.K. Hayman, Meromorphic Functions. Oxford, 1964.

[10] W.K. Hayman, Research Problems in Function Theory. 1967.

[11] J. Clunier and W.K. Hayman, Symposium on Complex Analysis, Canterbury, 1973. London Math. Soc., Lecture Series 12, 1974.

Department of Mathematics

East China Normal University

SHANGHAI

P.R. ChINA 